\title{
KAHBPS-1
}

\section{Absence of association between pretransplant serum soluble PD-1 level and prognosis following living donor liver transplantation in patients with hepatocellular carcinoma}

\author{
Shin HWANG*
}

Department of Surgery, Asan Medical Center, University of Ulsan College of Medicine, Seoul, Korea

Background: Programmed death protein 1 (PD-1) pathway is one of the most critical mechanisms in tumor biology of hepatocellular carcinoma (HCC). The study aimed to assess the prognostic influence of pretransplant serum soluble PD-1 (sPD-1) in patients undergoing liver transplantation for treatment of HCC.

Methods: Data from 229 patients with HCC who underwent living donor liver transplantation between January 2010 and December 2015 were retrospectively evaluated. Stored serum samples were used to measure sPD-1 concentrations.

Results: Overall survival (OS) and disease-free survival (DFS) rates were $94.3 \%$ and $74.5 \%$ at 1 year; $78.2 \%$ and $59.2 \%$ at 3 years; and $75.4 \%$ and $55.5 \%$ at 5 years, respectively. Prognostic analysis using pretransplant serum sPD-1 with a cutoff of $93.6 \mu \mathrm{g} / \mathrm{mL}$ (median value of the study cohort) did not have significant prognostic influence on OS $(p=0.69)$ and DFS $(p=0.26)$. Prognostic analysis using sPD-1 with a cutoff of $300 \mu \mathrm{g} / \mathrm{mL}$ showed similar OS $(p=0.46)$ and marginally lower DFS $(p=0.070)$. Combination of Milan criteria and sPD-1 with a cutoff of $300 \mu \mathrm{g} / \mathrm{mL}$ showed similar outcomes of OS and DFS in patients within and beyond Milan criteria. Multivariate analysis revealed that only Milan criteria was an independent prognostic for OS and DFS, but pretransplant sPD1 with a cutoff of $300 \mu \mathrm{g} / \mathrm{mL}$ did not become a prognostic factor.

Conclusions: The results of this study demonstrate that pretransplant serum sPD-1 did not show significant influences on post-transplant outcomes in patients with HCC. Further large-scale, multicenter studies are necessary to clarify the role of serum sPD-1 in liver transplantation recipients. 